\title{
Elevated IQGAP1 and CDC42 levels correlate with tumor malignancy of human glioma
}

\author{
XIAOBO CUI, LAIXIAO SONG, YUNFEI BAI, YAPING WANG, BOQIAN WANG and WEI WANG \\ Inner Mongolia Medical University Affiliated Hospital, Hohhot, Inner Mongolia 010050, P.R. China
}

Received May 12, 2016; Accepted December 12, 2016

DOI: $10.3892 /$ or.2016.5341

\begin{abstract}
IQGAP1 is a multifunctional scaffold protein involved in cell adhesion and cell migration. The abnormal expression of IQGAP1 widely exists in many cancers, but the combined biological roles of IQGAP1 and CDC42 in human glioma remain to be clarified. In this study, we investigated the associated expression level of IQGAP1, CDC42 and clinical significances in human glioma, as well as its biological functions in glioma progression. Our results revealed that IQGAP1 and CDC42 are frequently elevated in glioma tissues compared with their noncancerous counterparts, and a high expression of IQGAP1 and CDC42 correlates with tumor grades and poor overall survival of glioma patients. Moreover, the overexpression of IQGAP1 improves cell proliferation and migration ability of human glioma cells, whereas the knockdown of IQGAP1 by siRNA reduces cell growth and cell migration in vitro. These results suggest that IQGAP1, CDC42 and their interactions play important roles in human glioma carcinogenesis and progression.
\end{abstract}

\section{Introduction}

Glioma is the most common brain tumor, accounting for $80 \%$ of all primary brain and central nervous system malignancies (1). According as the World Health Organization (WHO) 2007 version based on the different histological tumor types, gliomas are classified as astrocytic, oligodendroglial, mixed oligoastrocytic, and ependymal glioma, with malignancy grade I, II, III, and IV (2). Gliomas are not sensitive to chemotherapy or radiotherapy, or the combination treatment of these methods. The prognosis of malignant glioma remains very poor, and the median overall survival of patients with glioblastoma is limited to approximately 16 months within clinical

Correspondence to: $\mathrm{Dr}$ Xiaobo Cui, Inner Mongolia Medical University Affiliated Hospital Otorhinolaryngology, 1 Tongdao North Street, Hohhot, Inner Mongolia 010050, P.R. China E-mail: nmcui2016@163.com

Key words: IQ motif containing GTPase-activating protein 1, CDC42, glioma, upregulation, cell migration trials $(2,3)$. Thus, it is necessary to find biomarkers and effective therapeutic targets for early diagnosis and improvement of the prognosis for glioma.

The IQ motif containing GTPase-activating protein 1 (IQGAP1) is a scaffold protein that regulates distinct cellular processes including actin dynamics, cell adhesion, cell motility, extracellular signals through interaction with cell adhesion molecules, several signaling molecules and cytoskeleton components (4-7). Many studies have revealed that IQGAP1 is upregulated in various human malignancies, such as colon cancer (8), lung cancer (9), hepatocellular carcinoma (10) and breast cancer (11). IQGAP1 plays a critical role in cancer cell invasion. The overexpression of IQGAP1 in colon cancer cells correlates with poor prognosis (12), and cell motility was increased by overexpressing IQGAP1 in breast cancer (13). Moreover, the upregulation of IQGAP1 has been found to be associated with poor prognosis of glioma and could be a potential prognosis marker (14).

As a scaffold protein, IQGAP1 interacts with multiple proteins to exert various roles in carcinogenesis. For example, IQGAP1 interacts directly with $\mathrm{RhoA} / \mathrm{C}$ to act as a regulator and pro-oncogenic effector of RhoA/C in breast cancer (13). Moreover, another study reported IQGAP1 can bind with $\beta$-catenin to promote liver cancer progression in vitro and in vivo (10). CDC42, one Rho family member, contributes to oncogenic transformation, invasion, and tumorigenesis (15). In breast cancer, the active CDC42 and RhoA trigger the interaction of IQGAP1 with exocyst subunits (16), which is required for matrix proteolysis and invasion of breast carcinoma cells. We focused on the association of IQGAP1 level with CDC42 expression in human glioma, and how two proteins collaborate to exert biological effects in glioma development.

In this study, we investigated the associated expression level and clinical significance of IQGAP1 and CDC42 in human glioma progression. We confirmed that IQGAP1 and CDC42 are frequently overexpressed in glioma tissues compared with their noncancerous counterparts, and high expression of IQGAP1 and CDC42 is associated with glioma grade and overall survival of glioma patients. Furthermore, our study discovered that the overexpression or knockdown of IQGAP1 in glioma cells has significant effects on cell proliferation and migration in vitro. Our results suggest that the elevated level of IQGAP1, CDC42 and their associations contribute to human glioma carcinogenesis and progression. 


\section{Materials and methods}

Cell culture. The human glioma cell lines including U87 and H4 were obtained from American Type Culture Collection (Manassas, VA), and U251 cells were obtained from the Type Culture Collection of the Chinese Academy of Sciences (Shanghai, China). All cells were cultured in DMEM medium supplemented with $10 \%$ fetal bovine serum (FBS) (16000044, Gibco), $100 \mathrm{U} / \mathrm{ml}$ penicillin, and $100 \mu \mathrm{g} / \mathrm{ml}$ streptomycin. Cells were incubated in a humidified atmosphere at $37^{\circ} \mathrm{C}$ with $5 \% \mathrm{CO}_{2}$.

Tissue sample collection. This study was approved by the Institutional Ethics Committee of the Affiliated Hospital of Inner Mongolia Medical University (Inner Mongolia, China). Thirty cases of human glioma tissues (HGTs) and their paired para-cancerous brain tissues (PBTs) were surgically resected in the Affiliated Hospital of Inner Mongolia Medical University with the patients' informed consent. The HGTs and their paired PBTs were immediately stored frozen in liquid nitrogen for further use.

Bioinformatics analysis for protein-protein interaction network. IQGAP1-interacting proteins were predicted based on the online human protein-protein interaction (PPI) datasets (http://www.hprd.org/). These protein interactions were summarized from literature studies, which have been widely validated by low-throughput and high-throughput experiments and applied in disease research (17). Cytoscape software was applied for visualization and analysis of PPI networks, which provides various plugins for different analyses. PPI networks are illustrated as graphs in Cytoscape with the nodes representing the proteins and the edges representing their interactions (18).

Expression plasmids, siRNAs and cell transfection. The original IQGAP1 cDNA (gi57242794) clone was ordered from Generay Biotechnology (Shanghai, China), and was sub-cloned into a pCMV6 plasmid to obtain a recombinant plasmid pIQGAP1, which was confirmed to be correct by DNA sequencing. Glioma U87 and U251 cells were respectively seeded on a 6-well plate for culture overnight, cells were transiently transfected with $2.5 \mu \mathrm{g}$ pIQGAP1 plasmids for one well using Lipofectamine 2000 (cat. no. 11668-019, Life Technologies) according to the manufacturer's instructions.

The IQGAP1-specific siRNA (siIQGAP1) based on literature (19) was synthesized by RiboBio Co., Ltd. (Guangzhou, China). The siIQGAP1 sequence, 5'-UUA UCG CCC AGA AAC AUC UUG UUG G-3'; and negative control oligonucleotides (NC) were 5'-UUC UCC GAA CGU GUC ACG U-3'. The U87 and U251 cells were seeded on a 6-well plate to incubate with $100 \mathrm{nM}$ siIQGAP1 for one well by the transfection reagent (INTERFER, Polyplus Transfection) following the manufacturer's protocols.

Western blotting. Total cellular protein was extracted with RIPA buffer (50 mM Tris base, $1.0 \mathrm{mM}$ EDTA, $150 \mathrm{mM} \mathrm{NaCl}$, $0.1 \%$ SDS, $1 \%$ Triton X-100, $1 \%$ sodium deoxycholate, $1 \mathrm{mM}$ PMSF). Protein samples were separated on $10 \%$ SDS-PAGE and transferred onto the PVDF membrane (Amersham
Biosciences, Amersham, UK) to detect protein expression level. The PVDF membrane was respectively incubated with IQGAP1 antibody (ab133490, Abcam, 1:1000) or CDC antibody (ab187643, Abcam, 1:10000) at $4^{\circ} \mathrm{C}$ overnight, followed by three 15 -min washes in PBS within $0.1 \%$ Tween-20. The membranes were then incubated with HRP-conjugated secondary antibodies at $37^{\circ} \mathrm{C}$ for $60 \mathrm{~min}$. Detection was performed with Western blot reagent ECL (Amersham Biosciences). Membranes were re-probed with mouse anti$\beta$-actin (sc-1616, Santa Cruz Biotechnology, Inc., Santa Cruz, CA, USA) for normalization of signal as a control.

Cell viability. Cell viability was measured using MTT assay. After IQGAP1 overexpression or knockdown for $48 \mathrm{~h}$, $3 \times 10^{3}$ cells/well were seeded in one 96-well plate in DMEM supplemented with 10\% FBS to incubate for another 24-48 h. In addition, $20 \mu 1$ of $5 \mathrm{mg} / \mathrm{ml}$ MTT solution (Sigma) was added to each well to incubate for $2-4 \mathrm{~h}$ at $37^{\circ} \mathrm{C}$, the formazan crystals were dissolved with $150 \mu \mathrm{l}$ dimethyl sulfoxide (Sigma). Absorbance was determined at $490 \mathrm{~nm}$ on Multiskan MK3 (Thermo Scientific, Rockford, IL, USA) immediately. Each assay was separately performed for three replicates and all experiments were repeated at least three times.

Transwell migration assay. After being transfected with pIQGAP1 plasmids or IQGAP1-specific siRNA for $48 \mathrm{~h}$, $1 \times 10^{4}$ cells in $100 \mu 1$ serum-free DMEM were seeded in the upper chamber of a Transwell (Millipore, 8-mm pore size), and the bottom of the chambers was filled with $800 \mu \mathrm{l}$ of medium containing 10\% FBS to culture another $24 \mathrm{~h}$. The migrated cells moved toward medium containing 10\% FBS. The remaining cells were fixed and stained with $1 \%$ crystal violet. Images were captured using an inverted microscope (Olympus), and the migrated cells were counted manually. The percentage of migration cells on the condition of IQGAP1 overexpression or knockdown was calculated by comparison with the control treatment with the empty plasmids or nontargeting siRNA.

Immunohistochemistry. Tissues were fixed with paraformaldehyde, embedded with paraffin, then cut into sections of $5 \mu \mathrm{m}$ thickness for immunohistochemistry (IHC) analysis mainly according to literature studies (20). The first antibody against IQGAP1 (ab133490, Abcam), CDC (ab187643, Abcam) was respectively used at a dilution of 1:100, 1:400. The second antibody was a biotinylated $\mathrm{IgG}$ for $40 \mathrm{~min}$ incubate at $37^{\circ} \mathrm{C}$. Tissue slices were visualized by the 3, 3'-diaminobenzidine solution, and cellular nuclei were slightly counterstained with hematoxylin. Substitution of the primary antibody with phosphate-buffered saline (PBS) was taken as a control for IHC. According to general evaluation standards (21), the staining intensity was scored as 0 (negative), 1 (weak), 2 (moderate) or 3 (strong). The extent of staining was monitored based on the percentage of positive tumor cells: 0 (negative), 1 (1-25\%), 2 (26-50\%), 3 (51-75\%) and 4 (76-100\%). The final score of 0 was defined as a negative expression (-); scores of 1-3 were accepted as a low/weak expression (+), and scores over 3 were defined as a high/strong expression (++). The intensity and percentage of positive cells were evaluated at least in five separate fields at a 400 -fold magnification. The IHC scores of 


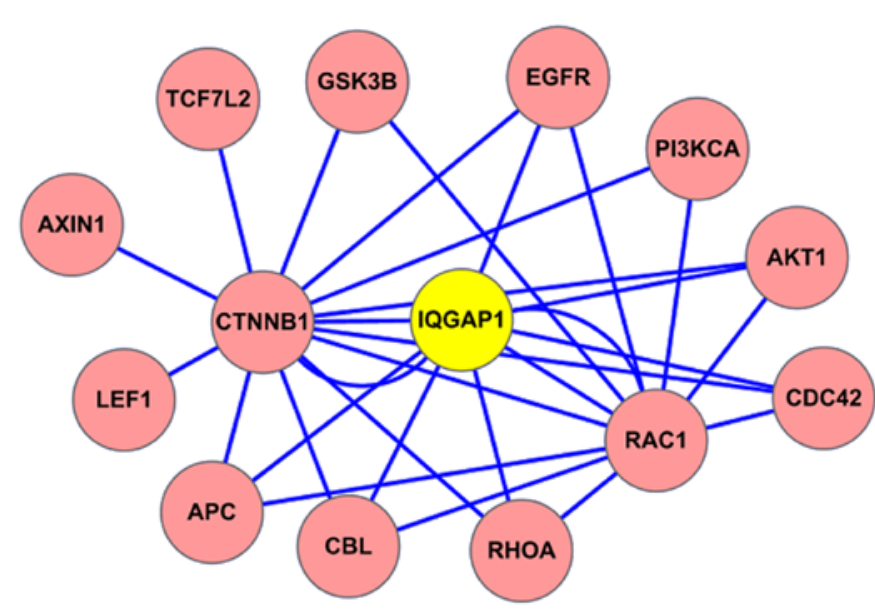

Figure 1. The interacting proteins with IQGAP1 analyzed based on HPRD database (http://www.hprd.org/). In the protein-protein interaction (PPI) map, IQGAP1 is located in the central position. The interacting partners are shown as red cycle. PPI networks are illustrated as graphs in Cytoscape with the nodes representing the proteins and the edges representing their interactions. The arrangement of nodes was applied to the 'Spring Embedded' layout in Cytoscape. HPRD, human protein-protein interactions database.

one tissue sample was respectively determined by two pathologists, and the final score for a tissue sample was calculated from the average value of the two sets of total scores. $\mathrm{P}<0.05$ was considered statistically significant.

Association analysis for protein expression and patient survival. The patient overall survival (OS) was evaluated using the Kaplan-Meier method (21). The 30 glioma patients were classified into two groups based on the protein expression level, including low IQGAP1/CDC42-expressing $(\mathrm{n}=8)$ and high IQGAP1/CDC42-expressing groups $(n=21)$. The group differences were assessed using the log-rank test. $\mathrm{P}<0.05$ was considered statistically significant.

Statistical analysis. All statistical analyses were performed using the SPSS software system (version 19.0; SPSS, Inc., Chicago, IL, USA). Statistical data were expressed as the mean \pm standard deviation (SD). $\mathrm{P}<0.05$ was considered to be statistically significant.

\section{Results}

IQGAP1 interacts with CDC42 by bioinformatics analysis. The interacting proteins with IQGAP1 were analyzed based on HPRD database online (http://www.hprd.org/). In the proteinprotein interaction (PPI) map, IQGAP1 located in the central position, and the protein CDC42 was shown to interact with IQGAP1 (Fig. 1). Of course, the known binding partners RhoA and Rac1 were also included within the IQGAP1 interacting protein network.

CDC42 level is linked with IQGAP1 expression in glioma cells. The endogenous expression levels of IQGAP1 and CDC42 are high in glioma U251, U87 and H4 cells (Fig. 2A). When IQGAP1 expression was elevated 1.95 times in U251 cells by transient transfection of pIQGAP1 plasmids for $48 \mathrm{~h}$, the relative level of CDC42 was also respectively increased to

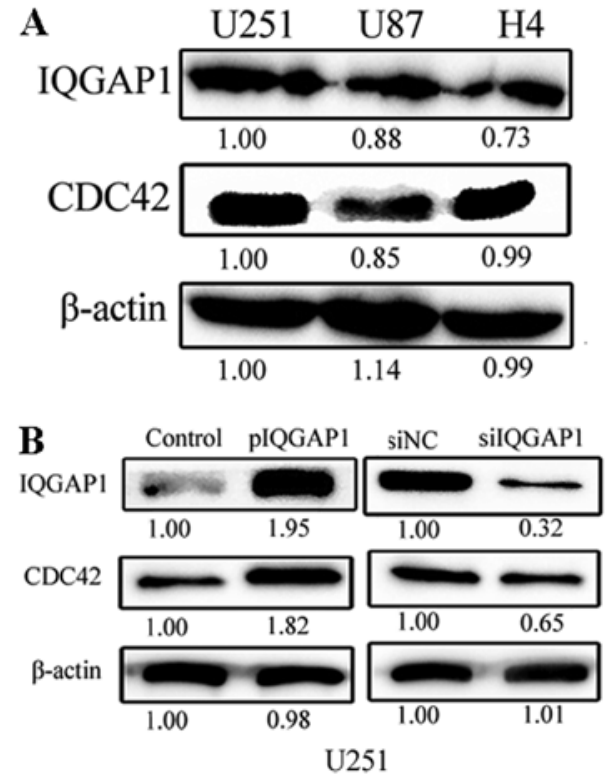

Figure 2. CDC42 level is linked with IQGAP1 expression in glioma cells. The expression level of IQGAP1, CDC42 was respectively measured in U251 and U87 cells at the native (A), overexpression (B) and knockdown conditions.

1.82-fold (Fig. 2B). When IQGAP1 expression was knocked down by siRNA treatment for $48 \mathrm{~h}, \mathrm{CDC} 42$ level was correspondingly decreased to 0.65 times in U251 cells. Similar co-expressing relationship between IQGAP1 and CDC42 was obtained in U87 cells (data not shown). Therefore, the expression of CDC42 is tightly linked with IQGAP1 level in glioma cells, which also indicates the two proteins interact with each other.

IQGAP1 overexpression promotes glioma cell growth and migration. In order to further investigate cellular biological influence of high IQGAP1 level, the gain- and loss-of-function studies were performed in glioma cells. We explored this protein biological effects on glioma U87 and U251 cells by overexpression or knockdown of IQGAP1 in these glioma cells.

The overexpression of IQGAP1 protein (Fig. 3A and B) significantly increased glioma cell proliferation (Fig. 3C and D) and cell migration (Fig. 3E and F) of U251 and U87 cells. For example, in pIQGAP1-transfected U251 cells, cell proliferation was increased by $37,68.7$ and $72.3 \%$ after transfection for 48-96 h compared with the vehicles (Fig. 3C). For IQGAP1-overexpressing U87 cells, cell growth curve was also obviously increased (Fig. 3D). Moreover, the quantity of cell migration was respectively increased to $1.84,1.34$ times in U251 and U87 cells which were transiently transfected with pIQGAP1 plasmids for $72 \mathrm{~h}$ (Fig. 3E and F).

On the contrary, knockdown of IQGAP1 significantly inhibited glioma cell proliferation rate and cell migration. When IQGAP1 was knocked down by siIQGAP1 for $72 \mathrm{~h}$ in U251 and U87 cells (Fig. 4A and B), cell growth was decreased by $30 \%$ (Fig. 4C and D), and cell migration number was decreased to over 2 times by comparison with the non-targeting siRNA groups (Fig. 4E and F). These results suggest that IQGAP1 downregulation greatly inhibits glioma cell proliferation and migration. 

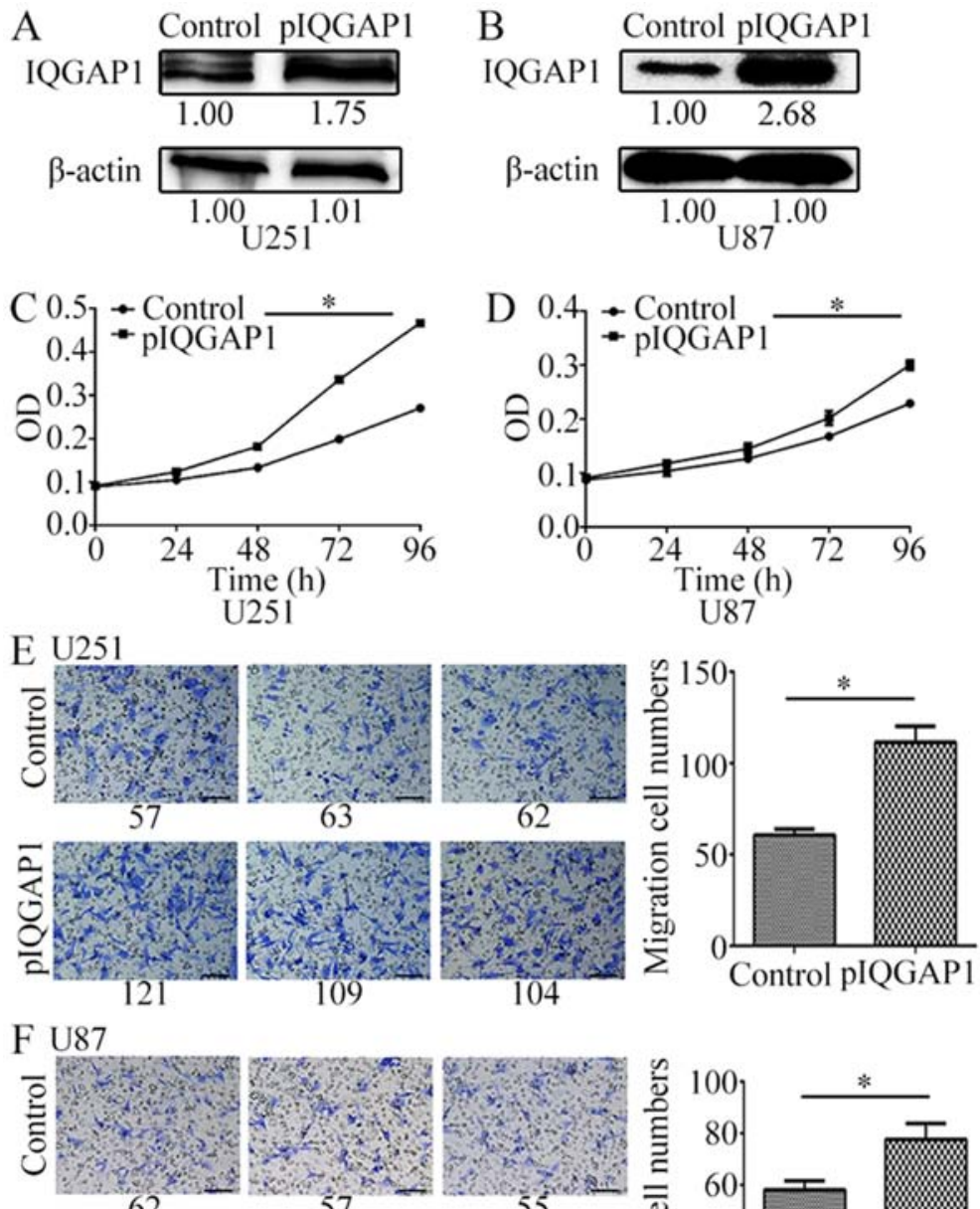

62
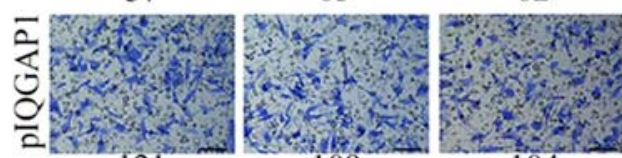

109

104
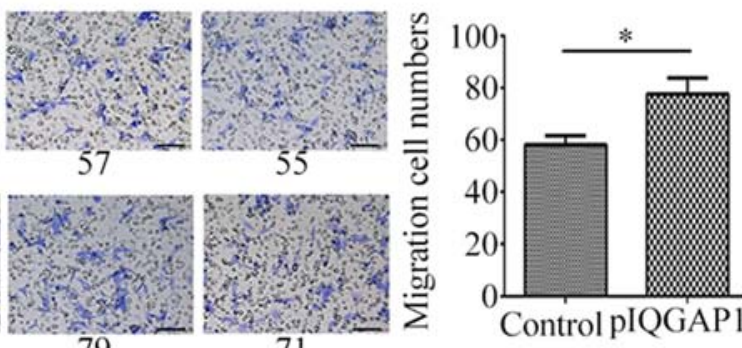

Figure 3. Overexpression of IQGAP1 promotes glioma cell proliferation and cell migration. Overexpression of IQGAP1 by transient transfection with pIQGAP1 plasmids in glioma U251 and U87 cells (A and B) enhanced cell proliferation (C and D), and cell migration (E and F) compared with the vehicles (control). Control, glioma cells transfected with the empty pCMV6 plasmids. pIQGAP1, glioma cells transiently transfected with IQGAP1-overexpressing plasmids. Scale bar represents $50 \mu \mathrm{m}$ (original magnification, $\mathrm{x} 400$ ). ${ }^{*} \mathrm{P}<0.05$.

Table I. The expression of IQGAP1 and CDC42 between HGTs and PBTs.

\begin{tabular}{lccccccc}
\hline & \multicolumn{3}{c}{ HGTs $(\mathrm{n}=30)$} & & \multicolumn{3}{c}{ PBTs $(\mathrm{n}=30)$} \\
\cline { 2 - 3 } Protein & Percentage & Average score & Expression level & & Percentage & Average score & Expression level \\
\hline \multirow{2}{*}{ IQGAP1 } & $100 \%(30 / 30)$ & $4.62 \pm 0.48$ & ++ & & $100 \%(30 / 30)$ & $1.30 \pm 0.16$ & + \\
& $26.7 \%(8 / 30)$ & $1.50 \pm 0.30$ & + & & $93.3 \%(28 / 30)$ & $1.11 \pm 0.09$ & + \\
& $73.3 \%(22 / 30)$ & $5.75 \pm 0.48$ & ++ & & $6.7 \%(2 / 30)$ & 4 & ++ \\
CDC42 & $100 \%(30 / 30)$ & $4.40 \pm 0.47$ & ++ & & $100 \%(30 / 30)$ & $2.37 \pm 0.19$ & + \\
& $30.0 \%(9 / 30)$ & $1.56 \pm 0.28$ & + & & $73.3 \%(22 / 30)$ & $1.82 \pm 0.12$ & + \\
& $70.0 \%(21 / 30)$ & $5.62 \pm 0.44$ & ++ & & $26.7 \%(8 / 30)$ & $3.88 \pm 0.18$ & ++ \\
\hline
\end{tabular}

Student's t-test, $\mathrm{P}<0.01$. HGTs, human glioma tissues; PBTs, para-cancerous brain tissues. Percentage: (specific case/total cases). Low expression $(+)$ : scores 1-3; high expression $(++)$ : scores $\geq 3$.

IQGAP1 and CDC42 are increased in human glioma tissues. The expression level of IQGAP1 and CDC42 was greatly elevated in human glioma tissues compared with their counterparts by IHC analysis (Fig. 5A). The average immunostaining 

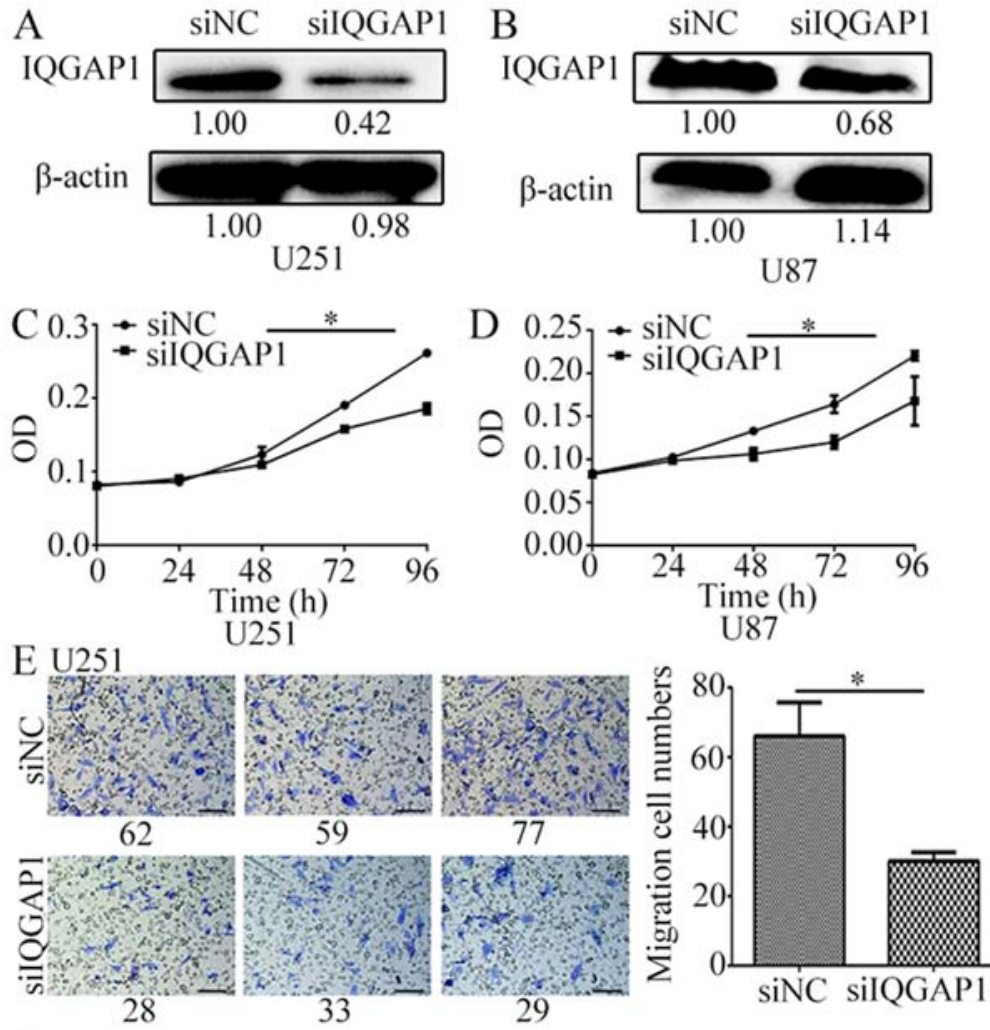

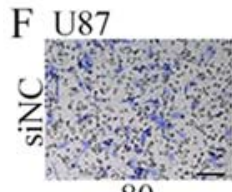

80

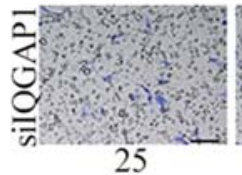

25

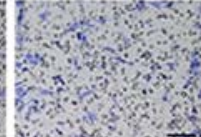

76

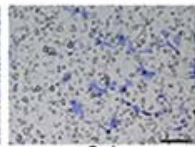

34
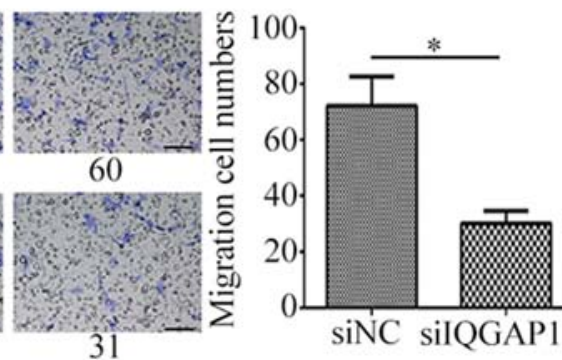

Figure 4. IQGAP1 knockdown decreases glioma cell growth and cell migration. Knockdown of IQGAP1 by siRNA in U251 and U87 cells (A and B) inhibited cell growth (C and D) and cell migration (E and F). siNC, glioma cells transfected with non-targeting control siRNA; si-IQ, glioma cells transfected with siRNA against IQGAP1. Scale bar represents $50 \mu \mathrm{m}$ (original magnification, $\mathrm{x} 400$ ). ${ }^{*} \mathrm{P}<0.05$.

score of IQGAP1, CDC42 was $4.62 \pm 0.48$ and $4.40 \pm 0.47$ in $30 \mathrm{HGTs}$, respectively, which was much higher than the average staining score $1.30 \pm 0.16,2.37 \pm 0.19$ in PBTs (Table I) $(\mathrm{P}<0.01)$. The IHC scores and clinical information for gliomas are provided in detail in Table II, and the IHC scores for PBTs are listed in Table III. Among the HGTs, more than $73.3 \%$ of glioma tissues (22 cases) showed a strong expression of IQGAP1 with scores $5.75 \pm 0.48$, and only 8 cases $(26.7 \%)$ had a weak expression of IQGAP1 with mean staining scores $1.14 \pm 0.26$ (Table I). While in 30 PBTs, IQGAP1 was usually detected with a lower expression level with an average staining score $1.30 \pm 0.16$. Only 2 cases showed strong expression with a score of 4 , most of PBTs (93\%) had low IQGAP1 expression scoring $1.11 \pm 0.09$. Similarly, 21 HGTs $(70 \%)$ had strong CDC42 level with scores $5.62 \pm 0.44$, which was higher than the frequency of $26.7 \%$ (8/30) with scores $3.88 \pm 0.18$ in PBTs. It was consistent with the IHC data that IQGAP1 and CDC42 had a strong expression in 3 randomly selected HGTs compared with their counterparts PBTs by western blot analysis (Fig. 5B).
Elevated IQGAPI and CDC42 expression are associated with glioma malignancy grade. Based on the activating roles for glioma cell growth and migration in vitro, we further discovered the clinical significance of the expression level of IQGAP1 and CDC42 for glioma development. The high expression level of IQGAP and CDC42 is positively associated with glioma malignancy (Table IV). The clinicopathological characteristics of glioma samples included patient gender, age and tumor TNM stage. It was clearly shown that a strong expression of IQGAP1, CDC42 existed in 22 advanced grade gliomas with TNM stages III-IV, with average IHC scoring $5.75 \pm 0.45$ and $5.48 \pm 0.45$. Whereas, a lower level of IQGAP1 and CDC42 was present in human gliomas with TNM stage I-II. This difference between protein expression level with tumor grade was obvious $(\mathrm{P}<0.01)$. However, the expression level of IQGAP1 and CDC42 has no linkage with glioma patient gender or age.

In conclusion, IQGAP1 and CDC42 show widely increased expression in glioma, and much higher expression levels of the two proteins are detected in high-grade glioma tissues. 
Table II. Protein IHC scoring and pathological information for human glioma tissues.

\begin{tabular}{|c|c|c|c|c|c|c|c|c|c|c|c|}
\hline \multirow[b]{2}{*}{ Case no. } & \multirow[b]{2}{*}{ Age } & \multirow[b]{2}{*}{ Gender } & \multirow{2}{*}{$\begin{array}{l}\text { TNM } \\
\text { stage }\end{array}$} & \multirow{2}{*}{$\begin{array}{l}\text { Survival time } \\
\text { (months) }\end{array}$} & \multirow{2}{*}{$\begin{array}{l}\text { Survival } \\
\text { state }\end{array}$} & \multicolumn{3}{|c|}{ Scoring of IQGAP1 } & \multicolumn{3}{|c|}{ Scoring of CDC42 } \\
\hline & & & & & & A & B & Average & A & B & Average \\
\hline 1 & 57 & Male & I & 35 & Survival & 0 & 0 & 0 & 2 & 2 & 2 \\
\hline 2 & 34 & Male & II & 32 & Survival & 1 & 1 & 1 & 1 & 1 & 1 \\
\hline 3 & 30 & Female & II & 36 & Survival & 2 & 3 & 2.5 & 2 & 3 & 2.5 \\
\hline 4 & 39 & Male & II & 24 & Death & 1 & 2 & 1.5 & 2 & 2 & 2 \\
\hline 5 & 58 & Female & II & 21 & Death & 3 & 2 & 2.5 & 1 & 1 & 1 \\
\hline 6 & 61 & Male & III & 25 & Death & 4 & 2 & 3 & 3 & 2 & 2.5 \\
\hline 7 & 30 & Female & III & 30 & Survival & 3 & 4 & 3.5 & 4 & 2 & 3 \\
\hline 8 & 51 & Male & III & 33 & Survival & 3 & 3 & 3 & 3 & 3 & 3 \\
\hline 9 & 36 & Female & III & 12 & Survival & 4 & 4 & 4 & 3 & 3 & 3 \\
\hline 10 & 57 & Female & III & 7 & Survival & 6 & 4 & 5 & 4 & 4 & 4 \\
\hline 11 & 73 & Female & III & 9 & Death & 3 & 3 & 3 & 4 & 4 & 4 \\
\hline 12 & 50 & Female & III & 10 & Survival & 4 & 6 & 5 & 6 & 3 & 4.5 \\
\hline 13 & 31 & Male & III & 11 & Survival & 3 & 4 & 3.5 & 2 & 4 & 3 \\
\hline 14 & 56 & Male & IV & 12 & Death & 6 & 4 & 5 & 4 & 4 & 4 \\
\hline 15 & 58 & Male & IV & 1 & Death & 8 & 9 & 8.5 & 8 & 8 & 8 \\
\hline 16 & 70 & Male & IV & 0.5 & Death & 8 & 8 & 8 & 9 & 6 & 7.5 \\
\hline 17 & 65 & Female & IV & 7 & Survival & 9 & 8 & 8.5 & 9 & 9 & 9 \\
\hline 18 & 65 & Female & I & 31 & Survival & 1 & 2 & 1.5 & 2 & 2 & 2 \\
\hline 19 & 61 & Male & II & 11 & Death & 2 & 2 & 2 & 0 & 0 & 0 \\
\hline 20 & 38 & Female & III & 3 & Survival & 4 & 4 & 4 & 4 & 4 & 4 \\
\hline 21 & 48 & Female & III & 2.2 & Death & 4 & 6 & 5 & 6 & 4 & 5 \\
\hline 22 & 63 & Male & III & 12 & Death & 4 & 6 & 5 & 4 & 6 & 5 \\
\hline 23 & 72 & Female & IV & 6 & Death & 6 & 9 & 7.5 & 6 & 8 & 7 \\
\hline 24 & 48 & Male & IV & 8 & Survival & 6 & 6 & 6 & 6 & 6 & 6 \\
\hline 25 & 56 & Female & IV & 9 & Survival & 6 & 6 & 6 & 6 & 8 & 7 \\
\hline 26 & 64 & Male & IV & 11 & Death & 6 & 8 & 7 & 6 & 9 & 7.5 \\
\hline 27 & 35 & Male & IV & 5 & Death & 9 & 9 & 9 & 8 & 8 & 8 \\
\hline 28 & 63 & Female & IV & 6 & Death & 9 & 8 & 8.5 & 6 & 9 & 7.5 \\
\hline 29 & 34 & Male & IV & 5 & Death & 9 & 8 & 8.5 & 8 & 8 & 8 \\
\hline 30 & 59 & Male & I & 13 & Death & 1 & 1 & 1 & 1 & 1 & 1 \\
\hline
\end{tabular}

A statistical analysis of the expression patterns revealed that there is a positive correlation between IQGAP1 and CDC42 expression $(\mathrm{p}<0.001)$ (Fig. 6).

IQGAPI/CDC42 expression inversely correlates with overall survival for glioma patients. Furthermore, the combined expression level of IQGAP1 and CDC42 was discovered to correlate with the overall survival of glioma patients. In order to determine the prognostic significance, 30 cases of glioma patients who have exact overall survival (OS) rates were grouped into two types, including a low (score <3) and high (score $>3$ ) protein expression level of IQGAP1/CDC42. Among 30 glioma patients, 21 and 8 cases were, respectively, included into the high and low expression level of IQGAP1/CDC42. The Kaplan-Meier estimates showed significant differences in OS rates between patients with a low level of IQGAP1/CDC42 and those with a high level of IQGAP1/CDC42 $(\mathrm{P}<0.05$ by the log-rank test; Fig. 7). The median overall survival was
12.0 months for 21 patients with high expression level of IQGAP1/CDC42, while the time was 30 months for 8 patients with low expression of IQGAP1/CDC42.

\section{Discussion}

As a hotspot of biological therapy, biomarker is an indicator of normal biological processes, pathogenic processes or a pharmacological response to a therapeutic intervention. In recent years, tumor biomarkers have been continuously reported due to the important roles in diagnosis, therapy and prognosis for cancer (22). As far as we know, potential glioma biomarkers have been widely screened through various multidisciplinary methods $(22,23)$, including gene chip, genome-wide approach, proteomics identification and molecular pathophysiology analysis (21). Although several biomarkers have been discovered to be important in the management of gliomas, including $1 \mathrm{p} 19 \mathrm{q}$ co-deletion, MGMT promoter methylation, BRAF and IDH1 
A

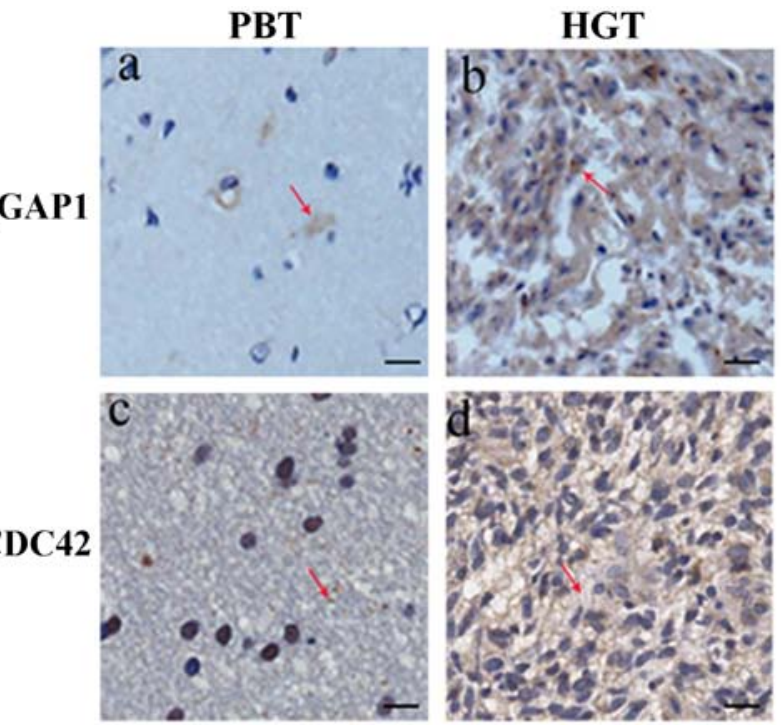

B

HGT 1 PBT 1
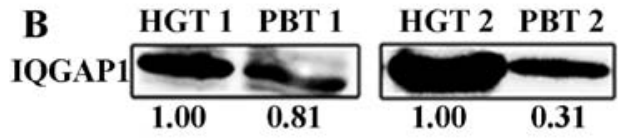

HGT 3 PBT 3
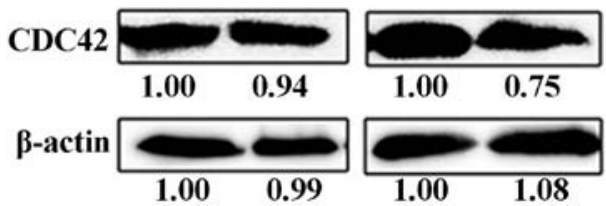

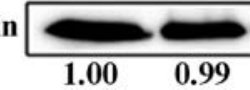

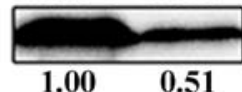
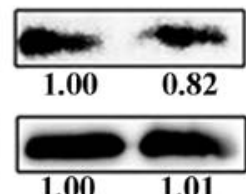

Figure 5. The expression level of IQGAP1 and CDC42 in HGTs and PBTs detected by IHC (A) and western blotting (B). The staining activity of IQGAP1, and CDC42 is, respectively, shown in PBTs (a and c) and HGTs (b-d). Scale bar represents $50 \mu \mathrm{m}$ with the original magnification, $\mathrm{x} 400$ HGTs, human glioma tissues. PBTs, para-cancerous brain tissues. The positive staining cells are shown with an arrow. (B). The expression of IQGAP1 and CDC42 was measured in three randomly selected glioma tissues and their counterparts.

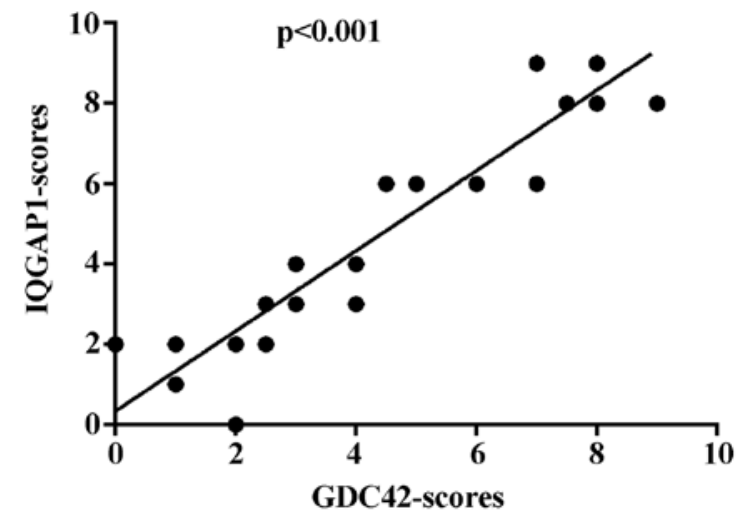

Figure 6. Expression correlation between IQGAP1 and CDC42 in glioma tissues. Statistical analysis of the expression patterns revealed that there was a positive expression correlation between IQGAP1 and CDC42 $(\mathrm{P}<0.001)$.

mutations, these potential biomarkers have certain limitations in clinical application (24). For example, it is known that the hypermethylation frequency of O(6)-methylguanine-DNA methyltransferase (MGMT) promoter varies widely in the different subtypes of glioma, and the methylation of MGMT appears to be a useful prognostic marker in the elderly patients with newly diagnosed glioblastoma (25). MGMT methylation

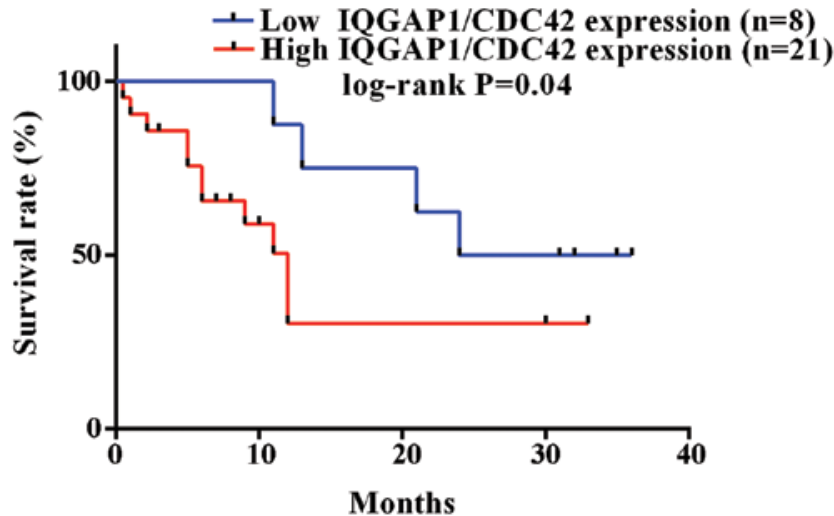

Figure 7. The expression of IQGAP1/CDC42 correlates with poor survival for glioma patients. The overall survival of glioma patients had obviously significant differences between patients with a high level of IQGAP1/CDC42 $(n=21)$ and a low level of IQGAP1/CDC42 $(n=8)(P<0.05$ by the log-rank test). Patients with a high level of IQGAP1/CDC421 had a worse postoperative overall survival.

Table III. Protein IHC scoring for para-cancerous brain tissues.

\begin{tabular}{|c|c|c|c|c|c|c|}
\hline \multirow[b]{2}{*}{ Case no. } & \multicolumn{3}{|c|}{ Scoring of IQGAP1 } & \multicolumn{3}{|c|}{ Scoring of CDC42 } \\
\hline & A & B & Average & A & B & Average \\
\hline 1 & 1 & 1 & 1 & 1 & 1 & 1 \\
\hline 2 & 1 & 1 & 1 & 2 & 2 & 2 \\
\hline 3 & 1 & 1 & 1 & 2 & 1 & 1.5 \\
\hline 4 & 0 & 0 & 0 & 1 & 1 & 1 \\
\hline 5 & 4 & 4 & 4 & 6 & 3 & 4.5 \\
\hline 6 & 1 & 3 & 2 & 4 & 4 & 4 \\
\hline 7 & 1 & 1 & 1 & 2 & 2 & 2 \\
\hline 8 & 1 & 1 & 1 & 3 & 2 & 2.5 \\
\hline 9 & 1 & 1 & 1 & 1 & 1 & 1 \\
\hline 10 & 1 & 1 & 1 & 3 & 2 & 2.5 \\
\hline 11 & 2 & 2 & 2 & 3 & 4 & 3.5 \\
\hline 12 & 1 & 1 & 1 & 1 & 2 & 1.5 \\
\hline 13 & 1 & 1 & 1 & 2 & 2 & 2 \\
\hline 14 & 1 & 1 & 1 & 3 & 1 & 2 \\
\hline 15 & 1 & 3 & 2 & 4 & 3 & 3.5 \\
\hline 16 & 0 & 0 & 0 & 2 & 2 & 2 \\
\hline 17 & 1 & 1 & 1 & 1 & 1 & 1 \\
\hline 18 & 1 & 1 & 1 & 2 & 2 & 2 \\
\hline 19 & 1 & 1 & 1 & 1 & 1 & 1 \\
\hline 20 & 4 & 4 & 4 & 6 & 3 & 4.5 \\
\hline 21 & 1 & 1 & 1 & 2 & 1 & 1.5 \\
\hline 22 & 1 & 1 & 1 & 2 & 3 & 2.5 \\
\hline 23 & 1 & 1 & 1 & 3 & 2 & 2.5 \\
\hline 24 & 1 & 3 & 2 & 4 & 4 & 4 \\
\hline 25 & 2 & 2 & 2 & 4 & 4 & 4 \\
\hline 26 & 1 & 1 & 1 & 2 & 2 & 2 \\
\hline 27 & 1 & 1 & 1 & 3 & 2 & 2.5 \\
\hline 28 & 1 & 1 & 1 & 2 & 4 & 3 \\
\hline 29 & 1 & 1 & 1 & 2 & 2 & 2 \\
\hline 30 & 1 & 1 & 1 & 2 & 2 & 2 \\
\hline
\end{tabular}


Table IV. Correlations of the expression of IQGAP1 and CDC42 in gliomas with clinical information.

\begin{tabular}{|c|c|c|c|c|c|c|c|}
\hline \multirow{2}{*}{$\begin{array}{l}\text { Clinicopathologic } \\
\text { variables }\end{array}$} & \multirow{2}{*}{$\begin{array}{l}\text { Number } \\
\text { (n) }\end{array}$} & \multicolumn{2}{|c|}{ Average score } & \multicolumn{2}{|c|}{ Expression level } & \multicolumn{2}{|c|}{ P-value } \\
\hline & & IQGAP1 & CDC42 & IQGAP1 & $\mathrm{CDC} 42$ & IQGAP1 & CDC42 \\
\hline \multicolumn{8}{|l|}{ Gender } \\
\hline Male & 16 & $4.50 \pm 0.76$ & $4.28 \pm 0.71$ & ++ & ++ & 0.8015 & 0.7927 \\
\hline Female & 14 & $4.75 \pm 0.59$ & $4.54 \pm 0.62$ & ++ & ++ & & \\
\hline \multicolumn{8}{|l|}{ Age } \\
\hline$<56$ & 13 & $4.35 \pm 0.66$ & $4.08 \pm 0.60$ & ++ & ++ & 0.6330 & 0.5576 \\
\hline$\geq 56$ & 17 & $4.82 \pm 0.70$ & $4.65 \pm 0.70$ & ++ & ++ & & \\
\hline \multicolumn{8}{|l|}{ TNM stage } \\
\hline I-II & 8 & $1.50 \pm 0.30$ & $1.44 \pm 0.29$ & + & + & $<0.001$ & $<0.001$ \\
\hline III-IV & 22 & $5.75 \pm 0.45$ & $5.48 \pm 0.45$ & ++ & ++ & & \\
\hline
\end{tabular}

$\mathrm{P}<0.05$ was considered statistically significant. P-value was calculated using Student's t-test. + , low expression; ++, high expression.

is well established as a prognostic/predictive marker for glioblastoma. However, it is not currently utilized widely in guiding patient management (24). It is necessary to establish more convenient and effective biomarkers for glioma diagnosis, treatment and prognosis based on the molecular basis of biomarker-mediated carcinogenesis.

By now, the overexpression of IQGAP1 has been reported to be associated with certain cancerous metastasis $(6,26-28)$. In our study, the correlation and biological effects between IQGAP1, CDC42 and glioma development have been clarified. IQGAP1 and CDC42 are widely upregulated in human glioma tissues, and their expression levels have a positive correlation with tumor malignancy. However, high expressions of IQGAP1 and CDC42 reversely correlate with glioma patient survival. Of course a more scale-up human glioma samples should be further verified for the associations of IQGAP1 level with glioma development and patient prognosis, which is very helpful for classifying and grading gliomas, as well as evaluating the potential predictive value based on the protein expression.

In addition, the contribution of the overexpression of IQGAP1 to glioma progression by promoting cell proliferation and cell migration needs clarification. According to our bioinformatics analysis of IQGAP1-interacting proteins based on human protein-protein interactions database (HPRD) (http:// www.hprd.org/), IQGAP1 with its interacting proteins involve in oncogenesis-associated signaling pathways (Fig. 1). The interacting proteins with IQGAP1 include CDC42, RAC1, RHOA, CTNNB1 ( $\beta$-catenin), APC, GSK3B, AXIN1, and EGFR partners. Among these interaction proteins, IQGAP1 mediates signaling by Rho family GTPases, including RAC1, RHOA and CDC42 to regulate cell-cell adhesion and cell migration (4). Our experimental data in vitro and in vivo demonstrate that upregulation of IQGAP1 and CDC42 improves cell proliferation and migration ability of human glioma cells, whereas the knockdown of IQGAP1 reduces cell growth and cell migration. Moreover, the protein-protein interactions of IQGAP1 and CDC42 enhance the oncogenic effects for glioma.

Targeting protein-protein interaction is a promising strategy to block cancer signal transduction $(29,30)$. Several new glioma therapeutic targets are currently being tested in clinical trials $(31,32)$, providing new approaches of targeted therapies for glioma. IQGAP1 is a scaffold protein by interacting with $\mathrm{CDC} 42$, which exerts a signal integrator to play crucial roles in affecting signal intensity and the specific cellular response to an extracellular cue, and their abnormal levels and changes contribute to glioma carcinogenesis and progression. Thus, it is interesting and valuable to further look for chemical small molecules or protein inhibitors targeting the interaction of IQGAP1 and CDC42, which is a novel strategy to develop new drugs for glioma.

\section{Acknowledgements}

This work was financially supported by the grants from the Natural Science Foundation of Inner Mongolia (grant no. 2014MS0856).

\section{References}

1. Dolecek TA, Propp JM, Stroup NE and Kruchko C: CBTRUS statistical report: Primary brain and central nervous system tumors diagnosed in the United States in 2005-2009. Neuro Oncol 14 (Suppl 5): v1-v49, 2012.

2. Brat DJ, Scheithauer BW, Fuller GN and Tihan T: Newly codified glial neoplasms of the 2007 WHO Classification of Tumours of the Central Nervous System: Angiocentric glioma, pilomyxoid astrocytoma and pituicytoma. Brain Pathol 17: 319-324, 2007.

3. Weller M, van den Bent M, Hopkins K, Tonn JC, Stupp R, Falini A, Cohen-Jonathan-Moyal E, Frappaz D, Henriksson R, Balana C, et al; European Association for Neuro-Oncology (EANO) Task Force on Malignant Glioma: EANO guideline for the diagnosis and treatment of anaplastic gliomas and glioblastoma. Lancet Oncol 15: e395-e 403, 2014.

4. Noritake J, Watanabe T, Sato K, Wang S and Kaibuchi K: IQGAP1: A key regulator of adhesion and migration. J Cell Sci 118: 2085-2092, 2005

5. Mataraza JM, Briggs MW, Li Z, Entwistle A, Ridley AJ and Sacks DB: IQGAP1 promotes cell motility and invasion. J Biol Chem 278: 41237-41245, 2003.

6. Johnson M, Sharma M and Henderson BR: IQGAP1 regulation and roles in cancer. Cell Signal 21: 1471-1478, 2009.

7. Watanabe T, Wang S, Noritake J, Sato K, Fukata M, Takefuji M, Nakagawa M, Izumi N, Akiyama T and Kaibuchi K: Interaction with IQGAP1 links APC to Rac1, Cdc42, and actin filaments during cell polarization and migration. Dev Cell 7: 871-883, 2004. 
8. Nabeshima K, Shimao Y, Inoue T and Koono M: Immunohistochemical analysis of IQGAP1 expression in human colorectal carcinomas: Its overexpression in carcinomas and association with invasion fronts. Cancer Lett 176: 101-109, 2002.

9. Miyoshi T, Shirakusa T, Ishikawa Y, Iwasaki A, Shiraishi T, Makimoto Y, Iwasaki H and Nabeshima K: Possible mechanism of metastasis in lung adenocarcinomas with a micropapillary pattern. Pathol Int 55: 419-424, 2005.

10. Jin X, Liu Y, Liu J, Lu W, Liang Z, Zhang D, Liu G, Zhu H, Xu N and Liang S: The overexpression of IQGAP1 and $\beta$-catenin is associated with tumor progression in hepatocellular carcinoma in vitro and in vivo. PLoS One 10: e0133770, 2015.

11. Jadeski L, Mataraza JM, Jeong HW, Li Z and Sacks DB: IQGAP1 stimulates proliferation and enhances tumorigenesis of human breast epithelial cells. J Biol Chem 283: 1008-1017, 2008.

12. Hayashi H, Nabeshima K, Aoki M, Hamasaki M, Enatsu S, Yamauchi Y, Yamashita Y and Iwasaki H: Overexpression of IQGAP1 in advanced colorectal cancer correlates with poor prognosis-critical role in tumor invasion. Int $\mathrm{J}$ Cancer 126: 2563-2574, 2010

13. Casteel DE, Turner S, Schwappacher R, Rangaswami H, Su-Yuo J, Zhuang S, Boss GR and Pilz RB: Rho isoform-specific interaction with IQGAP1 promotes breast cancer cell proliferation and migration. J Biol Chem 287: 38367-38378, 2012.

14. McDonald KL, O'Sullivan MG, Parkinson JF, Shaw JM, Payne CA, Brewer JM, Young L, Reader DJ, Wheeler HT, Cook RJ, et al: IQGAP1 and IGFBP2: Valuable biomarkers for determining prognosis in glioma patients. J Neuropathol Exp Neurol 66: 405-417, 2007.

15. Stengel K and Zheng Y: Cdc42 in oncogenic transformation, invasion, and tumorigenesis. Cell Signal 23: 1415-1423, 2011.

16. Sakurai-Yageta M, Recchi C, Le Dez G, Sibarita J-B, Daviet L, Camonis J, D'Souza-Schorey C and Chavrier P: The interaction of IQGAP1 with the exocyst complex is required for tumor cell invasion downstream of Cdc42 and RhoA. J Cell Biol 181: 985-998, 2008

17. Goel R, Muthusamy B, Pandey A and Prasad TS: Human protein reference database and human proteinpedia as discovery resources for molecular biotechnology. Mol Biotechnol 48 87-95, 2011.

18. Smoot ME, Ono K, Ruscheinski J, Wang PL and Ideker T: Cytoscape 2.8: New features for data integration and network visualization. Bioinformatics 27: 431-432, 2011.

19. Brandt DT, Marion S, Griffiths G, Watanabe T, Kaibuchi K and Grosse R: Dial and IQGAP1 interact in cell migration and phagocytic cup formation. J Cell Biol 178: 193-200, 2007.
20. Liang S, Xu Y, Shen G, Zhao X, Zhou J, Li X, Gong F, Ling B, Fang L, Huang $C$, et al: Gene expression and methylation status of 14-3-3sigma in human renal carcinoma tissues. IUBMB Life 60: 534-540, 2008

21. Lu W, Wang X, Liu J, He Y, Liang Z, Xia Z, Cai Y, Zhou L, Zhu $\mathrm{H}$ and Liang S: Downregulation of ARHGDIA contributes to human glioma progression through activation of Rho GTPase signaling pathway. Tumour Biol: Oct 10, 2016 (Epub ahead of print). doi: 10.1007/s13277-016-5374-6.

22. Liang S and Shen G: Biomarkers of glioma. In: Molecular Targets of CNS Tumors (ISBN: 978-953-307-736-9). Garami M (ed). Published by InTech Press, Rijeka, pp325-342, 2011.

23. Ma R, de Pennington N, Hofer M, Blesing C and Stacey R: Diagnostic and prognostic markers in gliomas - an update. $\mathrm{Br} \mathrm{J}$ Neurosurg 27: 311-315, 2013.

24. von Deimling A, Korshunov A and Hartmann C: The next generation of glioma biomarkers: MGMT methylation, BRAF fusions and IDH1 mutations. Brain Pathol 21: 74-87, 2011.

25. Gerstner ER, Yip S, Wang DL, Louis DN, Iafrate AJ and Batchelor TT: Mgmt methylation is a prognostic biomarker in elderly patients with newly diagnosed glioblastoma. Neurology 73: 1509-1510, 2009.

26. Dong P, Nabeshima K, Nishimura N, Kawakami T, Hachisuga T, Kawarabayashi $\mathrm{T}$ and Iwasaki $\mathrm{H}$ : Overexpression and diffuse expression pattern of IQGAP1 at invasion fronts are independent prognostic parameters in ovarian carcinomas. Cancer Lett 243: $120-127,2006$

27. Nakamura H, Fujita K, Nakagawa H, Kishi F, Takeuchi A, Aute I and Kato H: Expression pattern of the scaffold protein IQGAP1 in lung cancer. Oncol Rep 13: 427-431, 2005.

28. Balenci L, Clarke ID, Dirks PB, Assard N, Ducray F, Jouvet A, Belin MF, Honnorat J and Baudier J: IQGAP1 protein specifies amplifying cancer cells in glioblastoma multiforme. Cancer Res 66: 9074-9082, 2006

29. White CD, Brown MD and Sacks DB: IQGAPs in cancer: A family of scaffold proteins underlying tumorigenesis. FEBS Lett 583: 1817-1824, 2009.

30. Li H, Eishingdrelo A, Kongsamut $\mathrm{S}$ and Eishingdrelo $\mathrm{H}$ : G-protein-coupled receptors mediate 14-3-3 signal transduction. Signal Transduct Target Ther 1: 16018, 2016. doi: 10.1038/ sigtrans. 2016.18

31. Chi AS, Sorensen AG, Jain RK and Batchelor TT: Angiogenesis as a therapeutic target in malignant gliomas. Oncologist 14: 621-636, 2009.

32. Sanson M: Editorial review: Targets for glioma treatment: from bench to bedside. Curr Opin Oncol 20: 650-651, 2008. 